Numerical modelling of sequential droplet impingements

This article has been downloaded from IOPscience. Please scroll down to see the full text article.

2008 J. Phys. D: Appl. Phys. 41165303

(http://iopscience.iop.org/0022-3727/41/16/165303)

View the table of contents for this issue, or go to the journal homepage for more

Download details:

IP Address: 152.78.128.150

The article was downloaded on 29/04/2010 at 21:32

Please note that terms and conditions apply. 


\title{
Numerical modelling of sequential droplet impingements
}

\author{
S Kamnis ${ }^{1}, \mathbf{S} \mathbf{G u}^{1,3}$, T J Lu${ }^{2}$ and C Chen ${ }^{2}$ \\ ${ }^{1}$ School of Engineering Science, University of Southampton, Highfield, Southampton SO17 1BJ, UK \\ ${ }^{2}$ MOE Key Laboratory for Strength and Vibration, School of Aerospace Xi' an Jiaotong University, \\ Xi' an, 710049, People's Republic of China \\ E-mail:s.gu@soton.ac.uk and kamniss@aston.ac.uk
}

Received 23 April 2008, in final form 20 June 2008

Published 24 July 2008

Online at stacks.iop.org/JPhysD/41/165303

\begin{abstract}
During the process of thermal spray coating, molten powders are sprayed and deposited on substrates to generate protective coatings. It is essential to have a clear understanding of the physics of droplet impingement on the surface of substrates for better control of the generation of splats and the structure of coating. A numerical model is developed in this paper to simulate the dynamics of transient flow during the impingement process, including spreading, break-up, air entrapment and solidification. The computation is achieved using the technique of volume of fluid surface tracking within a fixed Eulerian structured mesh. The three-dimensional simulation is able to accurately give a demonstration of dynamic flow patterns such as the generation of fingers, satellite droplets and pores during impingement. The numerical model is validated with experimental data from the tin droplet measurement and excellent agreement is found between the simulation and the experiment.
\end{abstract}

(Some figures in this article are in colour only in the electronic version)

\section{Introduction}

The principle of droplet impingement on the solid surface has been applied to many engineering applications. For example, the purpose of thermal spray coating is to melt powder particles and project them onto substrates to produce protective coating. The quality of coating is a direct outcome of the impingement process in which individual splats are formed. Surface characterization techniques have been applied extensively to examine the structure of a coating. However, such an after-sprayed approach cannot provide direct information on the dynamic process of impingement and hence cannot enable more effective controls of coating. Mathematical modelling has become an important tool for process control and optimization in many engineering applications. A welldeveloped model can provide insight into the complicated physics by overcoming the technical constraints imposed by experiments.

The modelling of droplet impingement has been a subject of research for several decades. A good review about the existing models can be found in Kamnis and $\mathrm{Gu}$ [1]. Most

\footnotetext{
${ }^{3}$ Author to whom any correspondence should be addressed.
}

of the existing models are based on various assumptions which limit their predictability in engineering applications. The authors have been engaged in the development of more accurate numerical models to replace the many assumptions contained in the existing work. The most recent progress includes a two-dimensional (2D) single droplet impact model [1] incorporated with a number of improvements, such as a Marangoni stress boundary to solve the shear stress on the substrate surface as a function of temperature, additional heat transfer resistance in the governing equation for air entrapment and detailed thermophysical properties. The numerical methods have been successfully tested in 2D models and achieved excellent agreement with the experimental data of Shakeri and Chandra [2]. The success in modelling a single droplet in the 2D domain paves the way for computationally more extensive research such as droplet interactions.

The sequential droplet impingement is one of the typical phenomena of droplet interactions, and very limited work has been reported [3-5]. Simulation of sequential droplet impingement is challenging as the surface structure of the previously formed splats is complex and can only be represented by three-dimensional (3D) models. The demand for computation will be extremely high when very fine meshes 


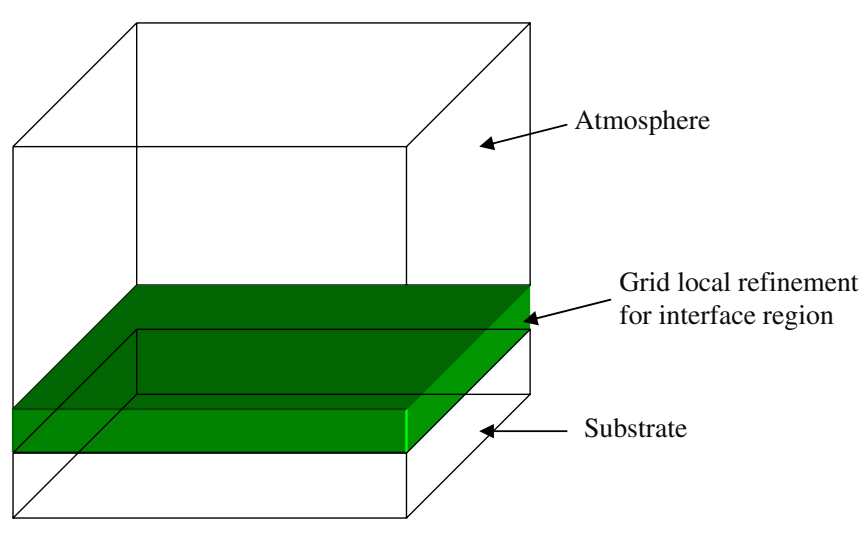

Figure 1. Computational domain.

are needed near the droplet-splat/droplet-substrate interfaces if the formation of air voids underneath the splat is considered. It is not surprising to find that the literature on the modelling of air entrapment underneath the splat is scarce due to the very high grid density required to capture such micro-structures. Instead, the focus has been put upon the final splat shape to simplify the formation process and reduce the cost of computation. A typical example of these computationally less expensive techniques is to apply a set of rules that specify the final splat shape as a function of droplet impact conditions. For instance, the models in [6-9] are based on the assumption that droplet spreading is influenced solely by the nearest previously deposited splat under it. A series of studies on the coating formation [10-13] indicate that coating porosity is related to numerous scenarios during the deposition including the instability and break-up of impinging droplets. The advance in computational methods and the availability of a high performance computing facility make it possible to examine the details of impingement without neglecting the complex effects by making various assumptions. The purpose of this work is to simulate two droplet impingements and to reveal the underlying physics of the process, which will throw light on the essential parameters and the phenomena for splat formation and pore generation. The information will help to improve the existing empirical models of dropletsplat interaction. The $3 \mathrm{D}$ simulation is a continuation of our previous work on 2D impingement models. Due to extensive computational costs, however, it is impossible to carry out a thorough parameter study for the 3D model as reported in [1].

\section{Numerical model}

The model of droplet impingement is set up in a computational domain (figure 1) which shows the local grid refinement applied to the interface region among the droplet, splat and substrate. A total of approximately 4 million computational cells are employed and the $7.5 \mathrm{~ms}$ dynamic simulation for two sequential droplets lasts for 1 month on an eight CPU Linux workstation. The numerical method used in this study is a segregated solution algorithm [14] with a control volume based technique. The pressure and velocity are coupled with the SIMPLE (semi-implicit method for pressure linked equations) algorithm $[15,16]$ which uses a guess-and-correct procedure for the calculation of the pressure on the staggered grid arrangement. The QUICK (quadratic upwind interpolation) scheme [17] is used for the discretization of the model equations. As a higher order scheme, the QUICK method can minimize false diffusion errors at the cost of the computational stability.

The droplet impingement model is developed and validated with the experimental measurement of tin droplets [2]. The material properties used in the current model can be found in [1]. Although pure metal is used in this simulation, the solidification model is developed for alloys and a temperature spectrum needs to be defined for the phase change. In this simulation, a small temperature range of $2 \mathrm{~K}$ is given for the tin droplet solidification. The numerical model works on the basis of the 3D incompressible laminar flow. Upon impingement, the transfer of heat is dominated by convection and conduction, and hence radiation from the droplet surface to the surroundings is negligible. The interaction between the droplet and the splat is achieved by releasing two sequential droplets from the same location to minimize the computational costs.

\section{Results and discussions}

\subsection{First droplet impingement}

3.1.1. Model validation. The accuracy of the previous 2D models has been validated with the available experimental data as reported in [1]. In a similar manner, the current 3D model is also validated. Figure 2 compares the computed dynamic impingement behaviour (left) with the photographic images taken by a high-speed camera [2] (right) at the same condition: a $2.1 \mathrm{~mm}$ diameter tin droplet at a temperature of $519 \mathrm{~K}$ landing with a velocity of $4 \mathrm{~m} \mathrm{~s}^{-1}$ on the $R=0.06 \mu \mathrm{m}$ rough stainless steel surface with a temperature of $298 \mathrm{~K}$. The results show that the droplet spreads radially along the substrate surface without breaking off from the edge under the current impact velocity and surface roughness at $0.1 \mathrm{~ms}$; the increase in instabilities around the edge as the droplet spreads initiates finger formation around the edge at $0.4 \mathrm{~ms}$ and subsequent stretching out of the fingers from the main body as the droplet expands. The generation of fingers is mainly due to the Rayleigh-Taylor instabilities which occur when the interface between two fluids of different densities accelerates; in this case, it is the interface between tin (density $7000 \mathrm{~kg} \mathrm{~m}^{-3}$ ) and air (density $1.2 \mathrm{~kg} \mathrm{~m}^{-3}$ ). It is apparent that excellent agreement is reached between the computation and the experiment, considering that exactly 28 fingers are found from the $2 \mathrm{~ms}$ prediction. A more quantitative comparison between the current numerical simulation and the experimental measurement [2] is made for the spreading factor in figure 3, which is consistent with the previous $2 \mathrm{D}$ modelling result [1].

3.1.2. Droplet dynamics. The velocity results shown in figure 4 illustrate that, upon impact, the liquid is forced to flow over the contact surface while the surface tension is comparatively small, with the maximum flow velocity achieved around the edge where all of the momentum is 

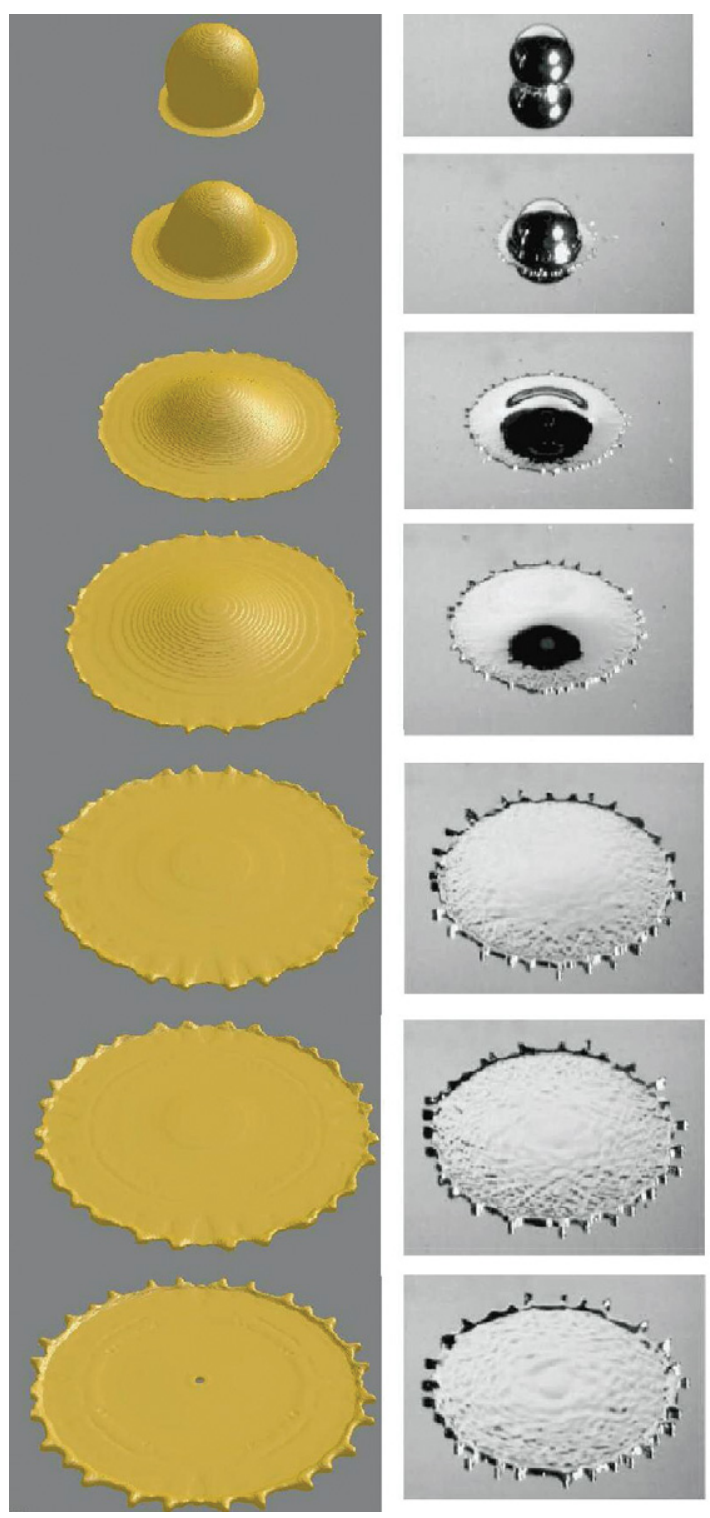

$0.1 \mathrm{~ms}$

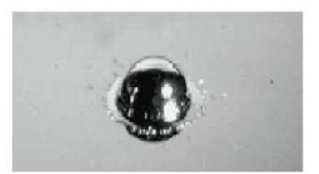

$0.2 \mathrm{~ms}$

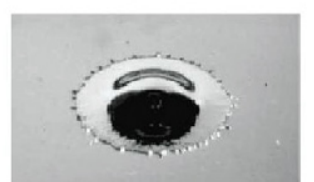

$0.4 \mathrm{~ms}$

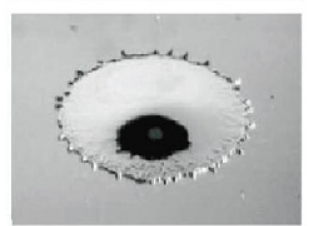

$0.6 \mathrm{~ms}$

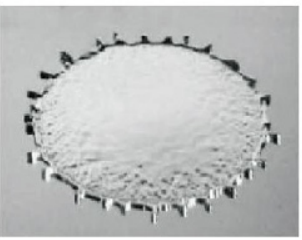

$1.1 \mathrm{~ms}$

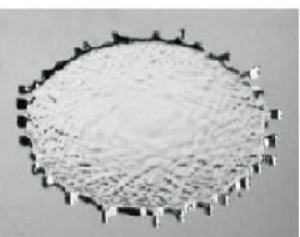

$1.4 \mathrm{~ms}$

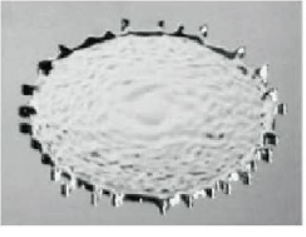

$2 \mathrm{~ms}$

Figure 2. Dynamic impact of single droplet impingement.

transferred to the radial direction and the cross-sectional area is small (the velocity at the edge is found to be $8 \mathrm{~m} \mathrm{~s}^{-1}$, $4 \mathrm{~m} \mathrm{~s}^{-1}$ higher than the impact velocity); more liquid gains high velocities as the droplet spreads at $0.4 \mathrm{~ms}$; later the flow slows down gradually when the surface tension around the edge and the centre becomes significant together with the development of solidification. The solidification plots shown in figure 5 demonstrate that solidification starts around the perimeter of the droplet right after the impact at $0.1 \mathrm{~ms}$ and expands as the droplet spreads at $0.4 \mathrm{~ms}$ (while the majority of the droplet is still in the full liquid state); the solid-liquid mixed area moves towards the centre as the droplet spreads out and no area is in the full liquid state at $2.0 \mathrm{~ms}$. In contrast, with the rapid mixing of solid and liquid regions, the change in the droplet temperature is not significant as shown in figure 6 . The temperature around the perimeter of the droplet starts to decrease below the solidus temperature $(504 \mathrm{~K})$ at $0.4 \mathrm{~ms}$; however, the majority of the droplet surface temperature remains close to the liquidus temperature $(506 \mathrm{~K})$ at $2.0 \mathrm{~ms}$.

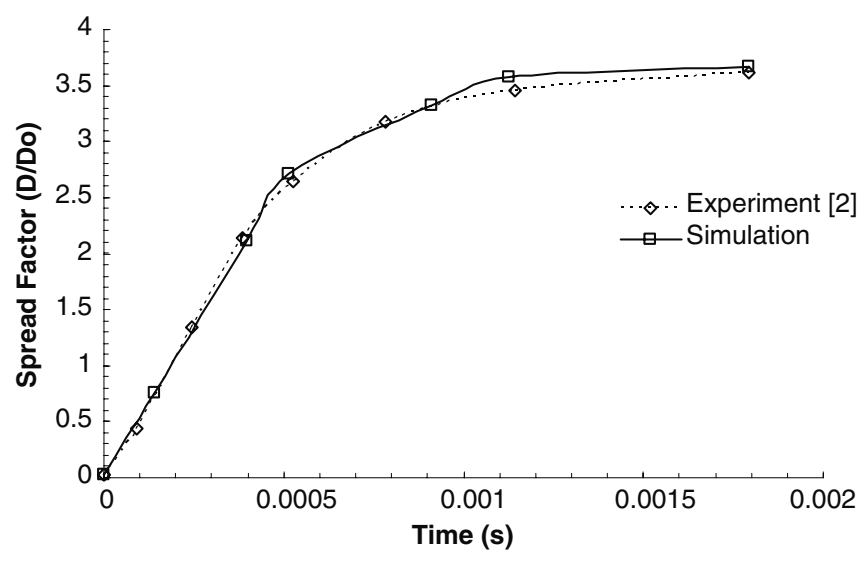

Figure 3. Comparison of the prediction of the spreading factor with the experiment.

3.1.3. Air entrapment. It is anticipated that air would be trapped between the droplet and the substrate during impingement. As a matter of fact, air involvement reduces the heat transfer rate between the droplet and the substrate, prolonging the solidification time. The 3D models are able to capture the development of air entrapment within the droplet during the impingement, as shown in figure 7. The density plots are used here as the large difference between tin and air densities makes the presence of air more identifiable. Air is trapped under the centre of the droplet upon impact and the void will remain in the centre during the course of time when the droplet develops into a splat. An array of voids is generated around the perimeter of the droplet at $0.4 \mathrm{~ms}$, which later moves outwards slightly as the droplet spreads. As a result, the air voids are located further away from the perimeter of the droplet at $2.0 \mathrm{~ms}$. The slow movement of air voids in comparison with spreading is attributed to the fact that solidification takes place faster on the contact surface between the droplet and the substrate, which prohibits void movement, while the droplet continues to spread with the down-flowing liquid from the main body. The generation of air entrapment is the interplay of many different parameters, such as the surface roughness and temperature, the impact velocity, the contact angle and the thermophysical properties of droplets. It is believed that the solidification process could play a considerable role in air entrapment. The effect of surface roughness on solidification and the consequent influence on air entrapment for a single droplet have been examined thoroughly in [1] and hence will not be repeated in the current computationally extensive 3D simulation.

\subsection{Second droplet impingement}

3.2.1. Droplet dynamics. The second droplet having the same conditions as the first one is released at $2.0 \mathrm{~ms}$ when the splat from the first droplet is yet to be fully solidified as described before. The interaction between the second droplet and the first splat is shown dynamically in figure 8 . Shortly after the second droplet lands on the partially solidified splat at $2.1 \mathrm{~ms}$, the impact force generates a ring of concavity around the periphery of the second droplet. The concavity ring 


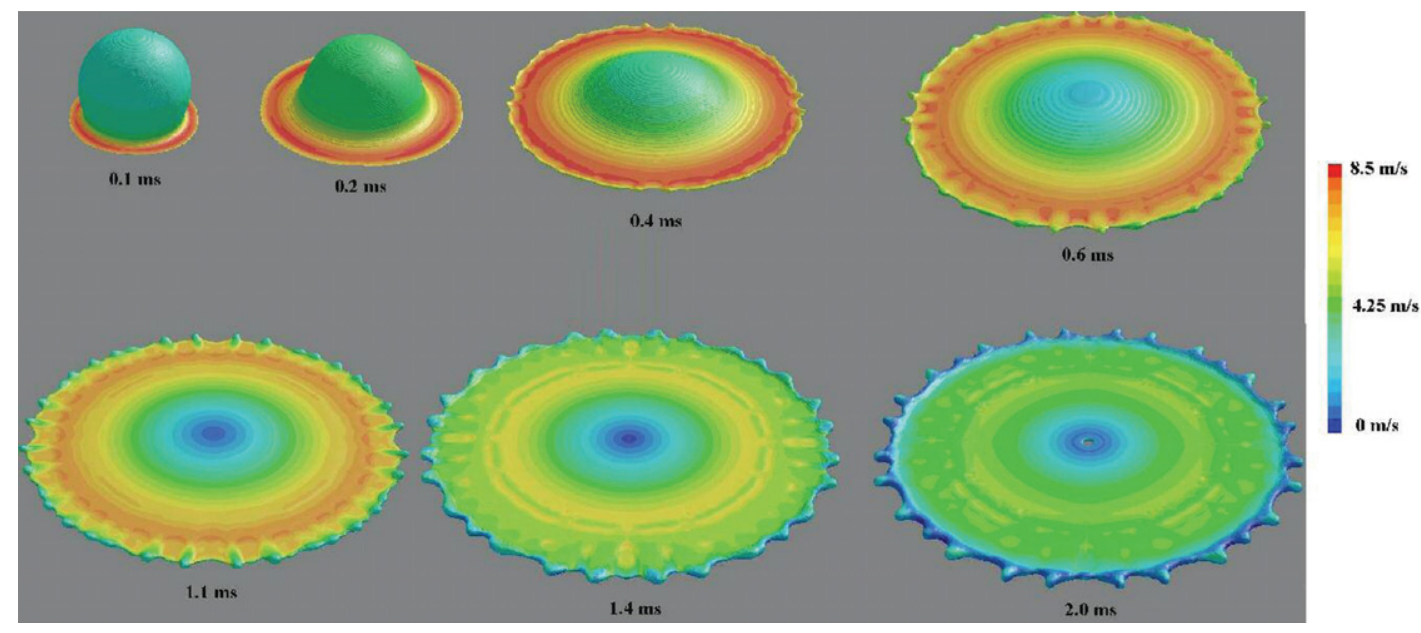

Figure 4. Surface velocity development during single droplet impingement.

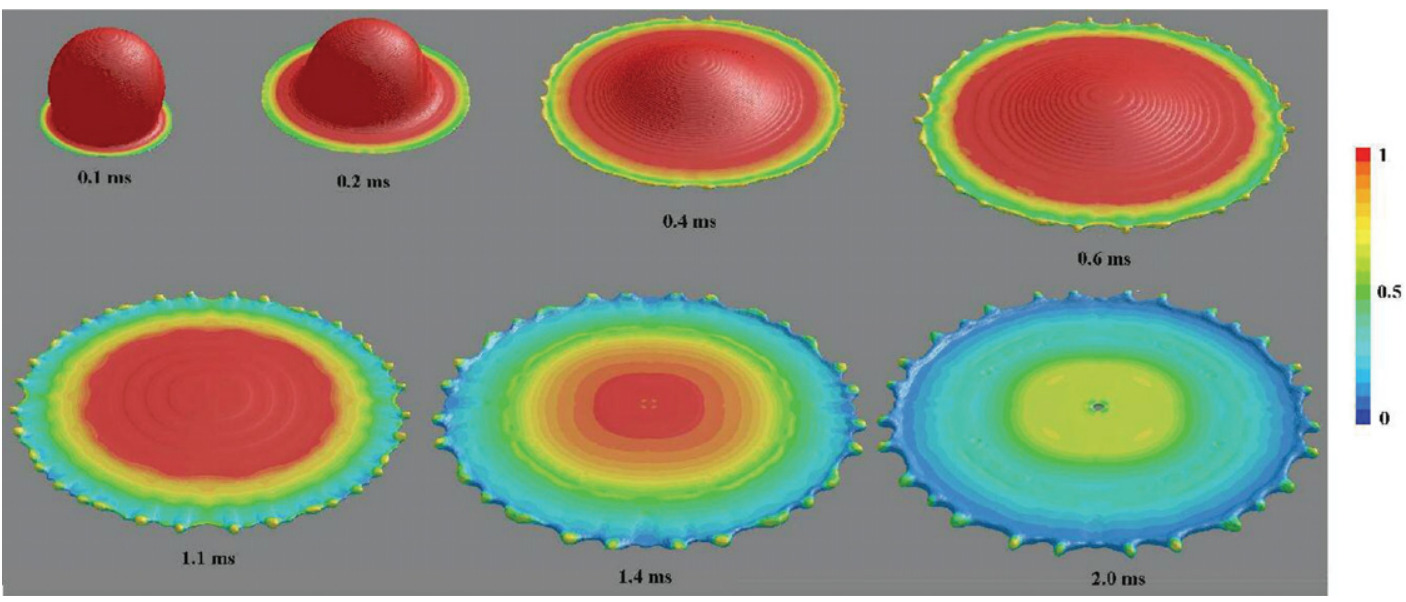

Figure 5. The solidification process of single droplet impingement.

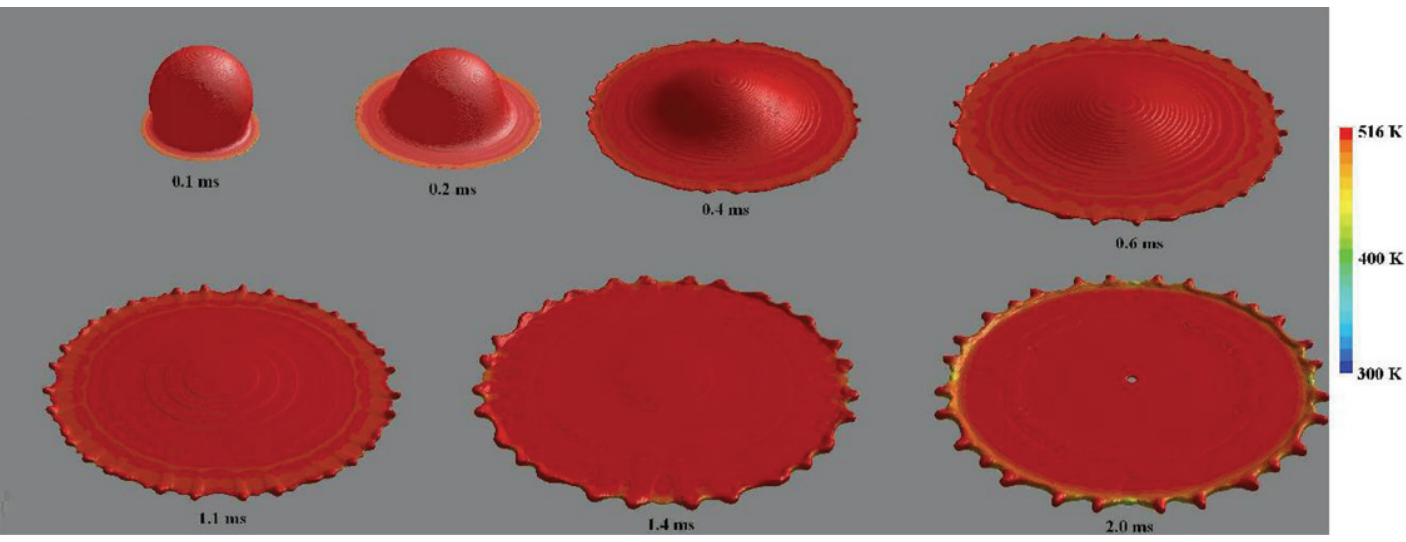

Figure 6. Surface temperature profiles during single droplet impingement.

expands and separates to a detached ring at $2.3 \mathrm{~ms}$; the upward expansion continues and two detached rings are observed at $2.5 \mathrm{~ms}$. Later the drastic expansion of the detached rings overgrows the size of the first splat and finally breaks down into satellite fragments scattering around the splat. The second splat mainly overlaps with the first splat and no noticeable geometric change is observed with the combined splats. The surface velocity results presented in figure 9 show high velocity profiles at the perimeter of the second droplet after the impact at $2.1 \mathrm{~ms}$ as the momentum is transferred from the vertical to the radial direction. The momentum gained by the detached rings enables them to outgrow the main body, decline gradually during expansion and eventually follow the free fall of the satellite fragments. The solidification and temperature results are shown in figures 10 and 11 , respectively. The second droplet has a high temperature profile which reduces the 


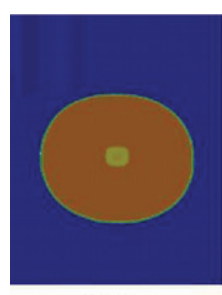

$0.1 \mathrm{~ms}$

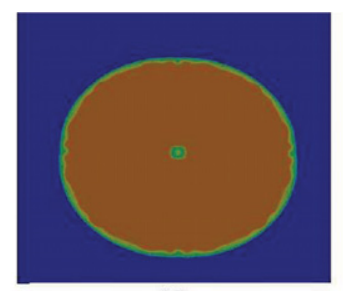

$0.2 \mathrm{~ms}$

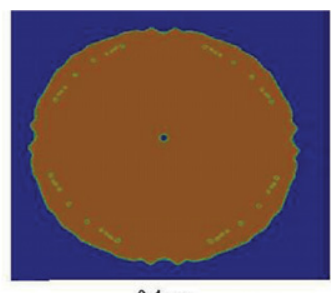

$0.4 \mathrm{~ms}$

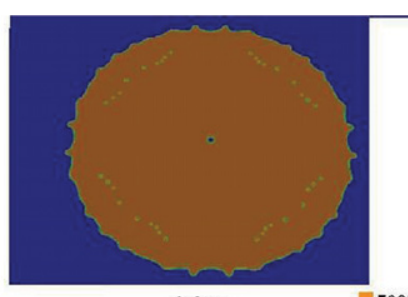

$0.6 \mathrm{~ms}$

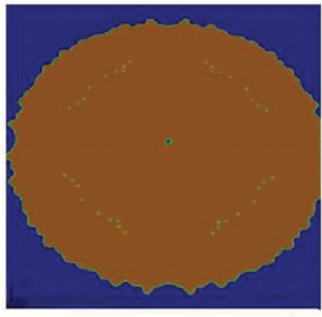

$1.1 \mathrm{~ms}$

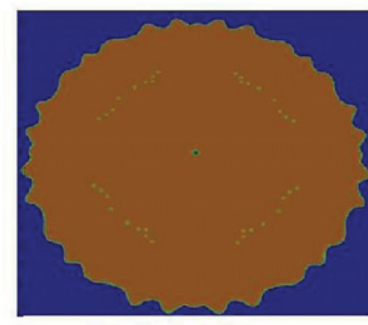

$1.4 \mathrm{~ms}$

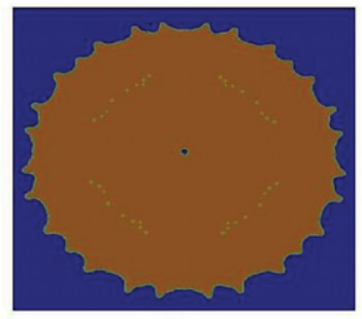

$2.0 \mathrm{~ms}$

Figure 7. Density plots for illustrating the air entrapment underneath the single droplet.
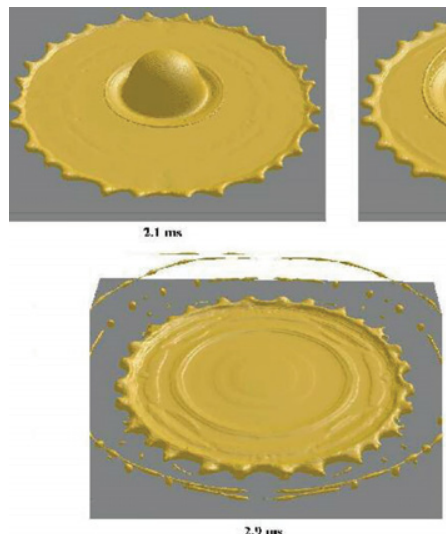

$2.9 \mathrm{mit}$
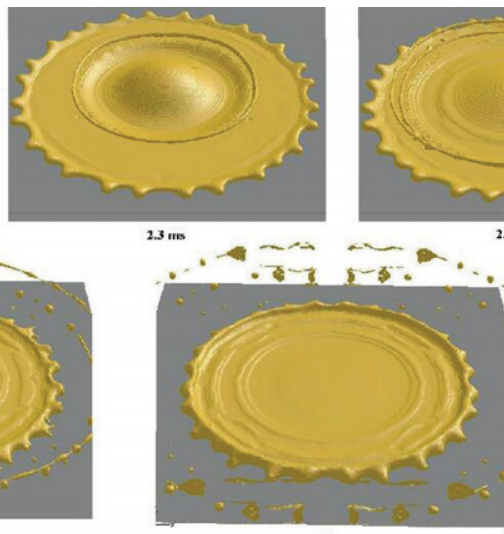

$3.1 \mathrm{~ms}$

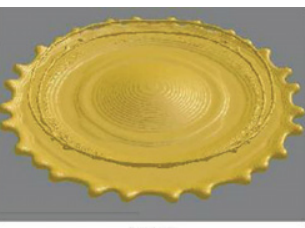

$2.5 \mathrm{~ms}$

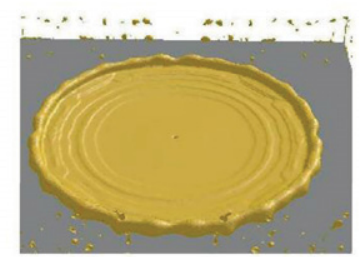

$7.5 \mathrm{~m}$

Figure 8. Dynamic impact of second droplet impingement.
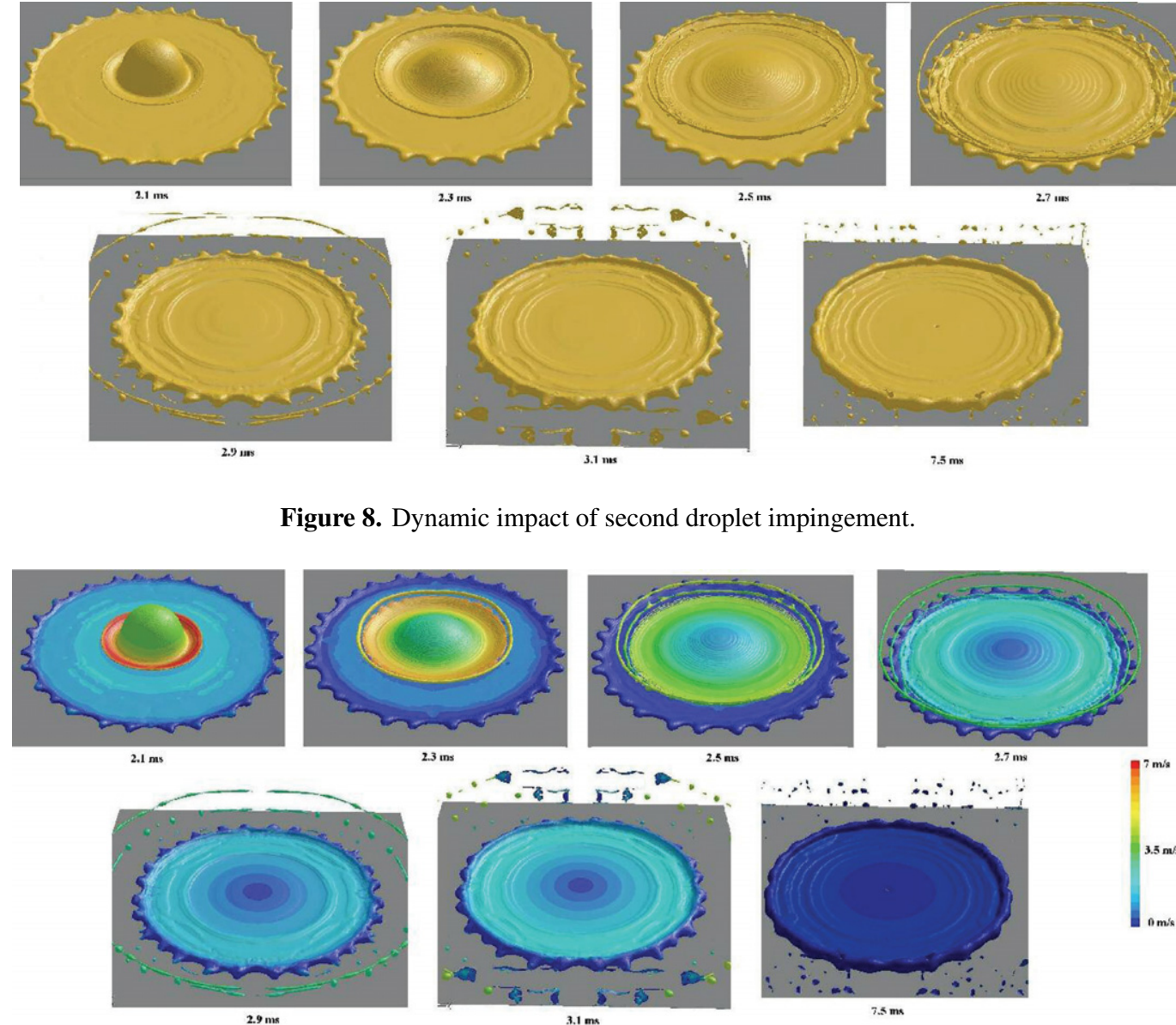

Figure 9. Surface velocity development during second droplet impingement.

solidification process within the first splat, while the combined splat reaches a full solid state at $7.5 \mathrm{~ms}$. The second splat is literally moulded inside the first splat, and the combined splats are thicker with more build-up around the edges.

3.2.2. Air entrapment. The numerically predicted development of air voids between the two splats is shown in figure 12. At the early stage of impact, there is no noticeable air entrapment till $2.7 \mathrm{~ms}$ when a few small voids are observed. The most significant development comes at $2.9 \mathrm{~ms}$ when the detached ring starts to break up, and a substantial number of small voids are developed within the second splat. Subsequently, the slight increase in air entrapment continues at $3.1 \mathrm{~ms}$ and stops as the droplets solidify. In comparison with 

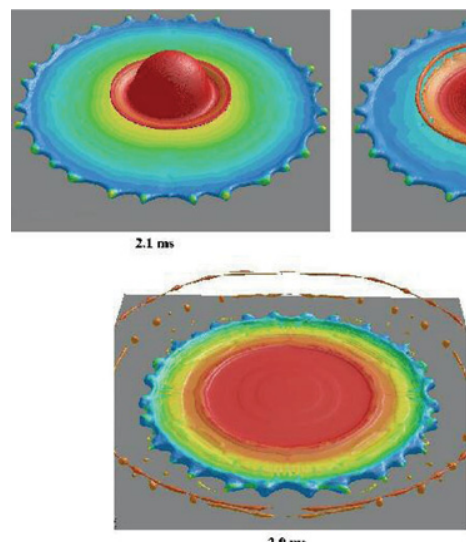
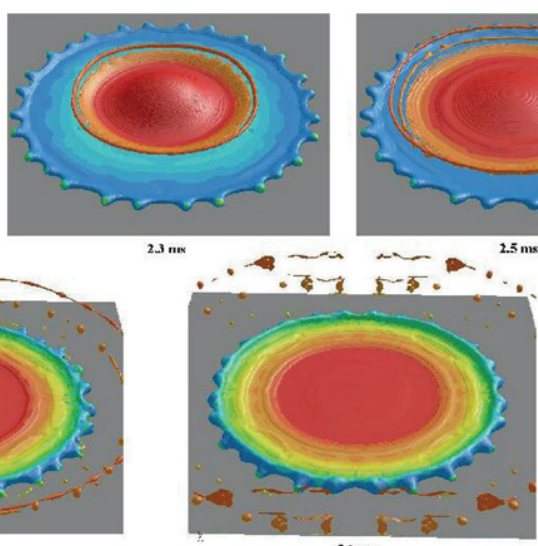

3.1
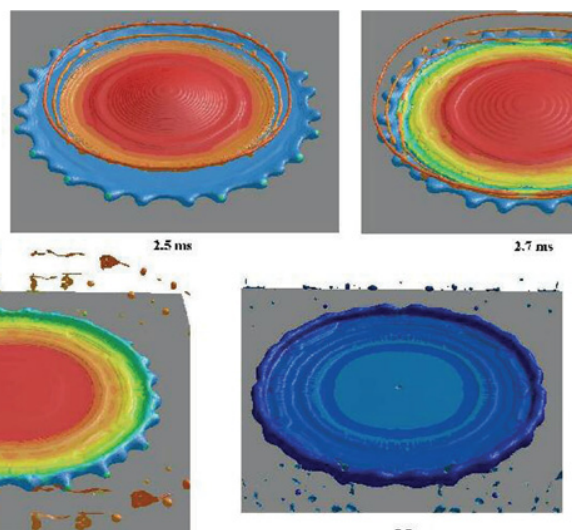

7.5 mo

Figure 10. The solidification process of second droplet impingement.

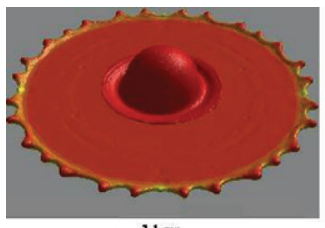

$2.10 \mathrm{~s}$

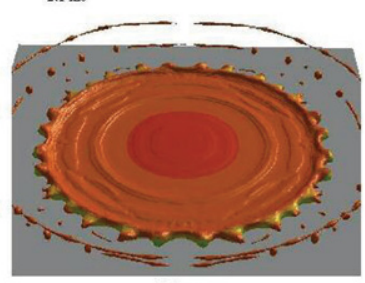

$2.9 \mathrm{~ms}$

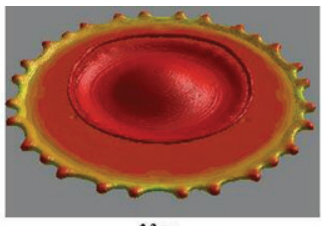

$2.3 \mathrm{~ms}$

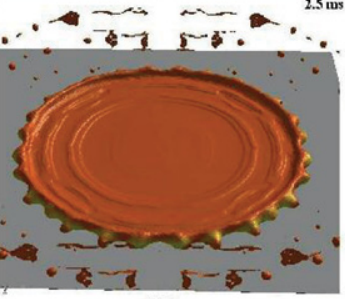

$3.1 \mathrm{~ms}$
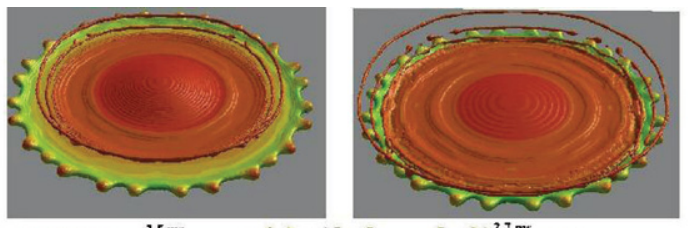

$516 \mathrm{~K}$

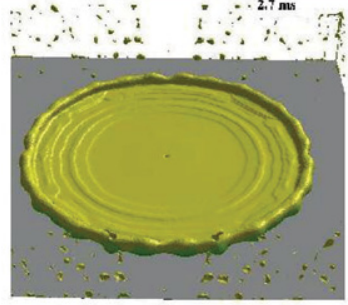

$7.5 \mathrm{~ms}$

Figure 11. Temperature profiles during second droplet impingement.

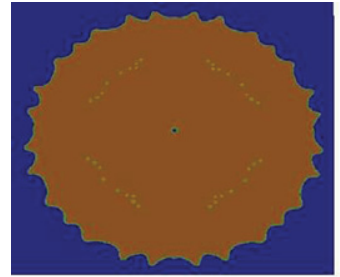

$2.1 \mathrm{~ms}$

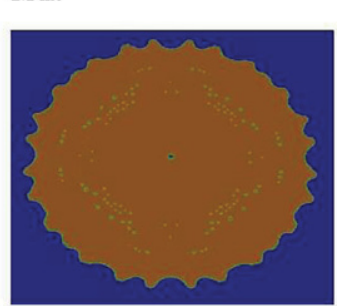

$2.9 \mathrm{~ms}$

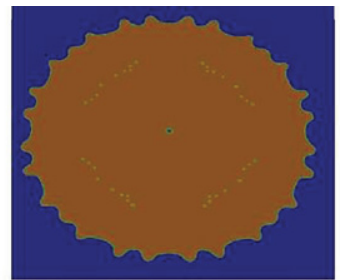

$2.3 \mathrm{~ms}$

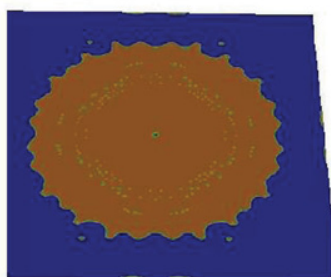

$3.1 \mathrm{~ms}$

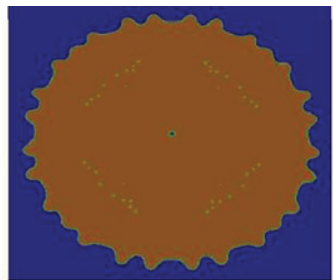

$2.5 \mathrm{~ms}$

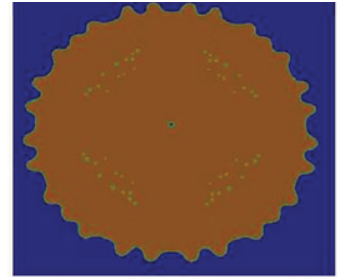

$2.7 \mathrm{~ms}$

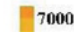

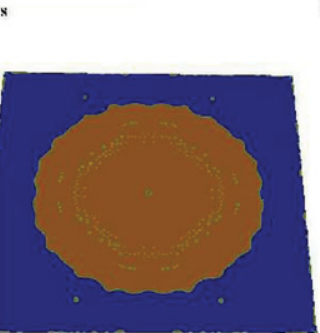

$7.5 \mathrm{~ms}$

Figure 12. Numerically predicted development of air voids between the two splats.

the first droplet results of figure 7, the appearance of air voids is delayed to $0.7 \mathrm{~ms}$ after the impact for the second droplet instead of $0.4 \mathrm{~ms}$ for the first droplet. A possible explanation could be that the first splat remains at a high temperature, with slow solidification taking place between the splats, whilst the solidification is faster between the interface of the droplet and the substrate during the first impingement. The number of air voids remains unchanged for the first splat, while there is a continued increase for the second splat. This phenomenon could be caused mainly by the complicated surface structure of the first splat, which also gives rise to the volatility of the second impingement. As the droplet becomes more unstable at $2.9 \mathrm{~ms}$ and the upwards moving detached ring starts to break, more air is trapped inside the splats. A slight increase in the air voids is further seen at $3.1 \mathrm{~ms}$ when both detached rings become fragmented. Thereafter no more entrapment occurs as 
the splats become fully solidified at $7.5 \mathrm{~ms}$. It is apparent that the air entrapment is more pronounced during the interaction of droplets.

\section{Conclusions}

A 3D numerical simulation is carried out to investigate the sequential tin droplet impingements on a stainless steel substrate. The numerical model is built upon our previous 2D development using the volume of fluid (VOF) methods by dynamically tracking the impact, spreading, solidification, splashing and air entrapment. Important 3D characteristics of the impingement process are revealed, including the accurate prediction of fingers in comparison with the experimental observation and the development of air voids within the splats. The results indicate that the surface structure of the previously generated splats give rise to more volatility of the droplet break-up during the impingement and increase the extent of air entrapment; on the other hand, the newly arrived splat tends to be moulded into the existing splat. With the growing availability of a high performance computing facility, the work presented in this paper demonstrates that it is possible to simulate the complex coating formation process using this numerical model.

\section{Acknowledgments}

The authors gratefully acknowledge the financial support from the UK DTI Technology Programme (TP/J3019E), the UKChina Fellowships for Excellence Programme provided by the UK DIUS, the National 111 Project of China (B06024), the National Natural Science Foundation of China (10572111, 10632060) and the National Basic Research Program of China (2006CB601202).

\section{References}

[1] Kamnis S and Gu S 2005 Numerical modelling of droplet impingement J.Phys. D: Appl. Phys. 38 3664-73
[2] Shakeri S and Chandra S 2002 Splashing of molten tin droplets on a rough steel surface Int. J. Heat Mass Transfer 45 4561-75

[3] Kang B, Zhao Z and Poulikakos D 1994 Solidification of liquid metal droplets impacting sequentially on a solid surface Int. J. Heat Mass Transfer 116 436-45

[4] Kang B, Waldvogel J and Poulikakos D 1995 Remelting phenomena in the process of splat solidification J. Mater Sci. 30 4912-25

[5] Ghafouri-Azar R, Shakeri S, Chandra S and Mostaghimi J 2003 Interactions between molten metal droplets impinging on a solid surface Int. J. Heat Mass Transfer 46 1395-407

[6] Ghafouri-Azar R, Mostaghimi J, Chandra S and Charmchi M 2003 A stochastic model to simulate formation of a thermal spray coating J. Therm. Spray Technol. 12 53-69

[7] Cirolini S, Harding J H and Jacucci G 1991 Computer simulation of plasma-sprayed coatings: I. Coating deposition model 48 137-45

[8] Ferrari M, Harding J H and Marchese M 1991 Computer simulation of plasma-sprayed coatings: II. Effective bulk properties and thermal stress calculations Surf. Coat. Technol. 48 147-54

[9] Kanouff M P, Nieser R A and Roemer T J 1998 Surface roughness of thermal spray coatings made with off-normal spray angles J. Therm. Spray Technol. 7 219-28

[10] McPherson R 1989 A review of microstructure and properties of plasma sprayed ceramic coatings Surf. Coat. Technol. 39-40 173-81

[11] Safai S and Herman H 1977 Microstructural investigation of plasma sprayed aluminum coatings Thin Solid Films 45 295-307

[12] Cai W D and Lavernia E J 1997 Modeling of porosity during spray forming Mater. Sci. Eng. A 226-228 8-12

[13] Fukanuma H 1994 A porosity formation and flattening model of an impinging molten particle in thermal spray coatings J. Therm. Spray Technol. 3 33-44

[14] Van Doormal J P, Raithby G D and McDonald B H 1987 The segregated approach to predicting viscous compressible fluid flows ASME J. Turbomach. 109 268-77

[15] Peric M 1990 Analysis of pressure-velocity coupling on non-orthogonal grids Numer. Heat Transfer B: Fundam. 17 63-82

[16] Raithby G D and Schneider G E 1979 Numerical solution of problems in compressible fluid flow: treatment of the velocity-pressure coupling Numer. Heat Transfer 2 417-40

[17] Leonard B P 1995 Order of accuracy of QUICK and related convection-diffusion schemes Appl. Math. Modelling 19 640-53 